\title{
USI TRASLATI DI TERMINI ASTRONOMICI. BOLIDE NELLA STAMPA ITALIANA E SPAGNOLA DEL XX SECOLO
}

\author{
Paola CAPponi \\ Universidad Pablo de Olavide (Sevilla)
}

\begin{abstract}
Resumen: En este artículo se realiza un análisis cuantitativo y cualititativo del uso de palabras técnicas, más en concreto de las palabras bolide y bólido, en la prensa italiana y española en el siglo XX. El objetivo es observar en qué medida estas palabras han ido adquiriendo o perdiendo sentidos figurados. El estudio permite reconstruir un proceso de ampliaciones y reducciones semánticas que si por un lado pone de manifiesto una progresiva tendencia a tecnificar, por otro lado insiste en la explotación del poder alusivo de la palabra.
\end{abstract}

Palabras clave: lexicon, press, astronomy, figurative sense, bolide.

Abstract: This article presents a quantitative and qualitative analysis of the use of the technical terms bolide y bólido as used in $20^{\text {th }} \mathrm{c}$. Italian and Spanish press, respectively. The aim of the study is to determine to what extent figurative senses of these words have either evolved or been lost. A process of semantic expansions and reductions will be reconstructed. This reconstruction highlights the progressive tendency to technicalize these words together with a tendency to exploit their allusive power.

Keywords: léxico, prensa, astronomía, sentido figurado, bólido.

Gridai, rapito sopra me: Vedeste?

Ma non v'era che il cielo alto e sereno.

(G. Pascoli, Bolide)

\section{TECNICISMI ASTRALI}

Nell'introduzione a Medieval Vierws of the Cosmos Evelyn Edson e Emilie Savage-Smith scrivono:

Once upon a time the universe had meaning. In place of our modern idea of formless, endless space, scattered sparsely and randomly with stars, planets, asteroids, black holes, pulsars, and quasars, there was a tightly structured, hierarchical system centred around the earth and the human race. At every level was found a moral lesson for humanity and a satisfying metaphor for 
the nature of God $[\ldots]$. All had its beginning in the divine command, 'Let there be light!', instead of the undignified big bang. Not only was thus system morally and spiritually meaningful, it was reinforced by simple observation and common sense. ${ }^{1}$

Gli oggetti che popolano il cielo oggi, che sfilano nell'elenco delle due autrici, si ritrovano nel Sonetto astrale di Edoardo Sanguineti:

pulsano pulsar con forti pulsioni:

ecco a voi quasar, quasi stelle vive:

collassano assai dense, per pressioni

che imbucano per sempre, in nere rive:

così forse è: facelle in evezioni, sciami di nebulose fuggitive, supergiganti, code in librazioni, variabili cefeidi recidive:

protuberanze, e getti, e radiazioni

corpuscolari, eclissi comprensive

di pieni pianetini e pianetoni,

aurore ipercompresse in somme stive:

oh, chiare notti gravitazionali,

mie fragili scintille zodiacali!

(10 ottobre 2008)

L'accostamento tra i due diversi testi ben si presta ad introdurre, anzi a porre la basi, dell'analisi che qui si propone. In entrambi si rintraccia un catalogo di oggetti celesti dai nomi nuovi. La sequenza crea distanza, raffredda, quasi gela. L'universo pare svincolarsi dall'umano (e dal divino), chiuso in una teca di parole senza connessioni visibili, quindi elencate. Lo esprimono le due autrici citando la perdita di significato del cosmo, la perdita del rimando morale, di una "satisfying metaphor for God", e con la citazione del big bang che segna quasi foneticamente la frattura con il più rassicurante "Let there be light!". Le autrici partono dalla perdita di significato («the universe had meaning», «system morally and spitritually meaningful») e legano poi questo significato, da un lato, alla struttura gerarchizzata e morale e, dall'altro, alla possibilità di osservazione a occhio nudo e al senso comune («simple observation and common sense»). A questa rassicurante struttura, quasi tangibile, e profondamente umana, si contrappone un cosmo definito quasi in absentia («formless», «endless space») di cui solo si conoscono gli oggetti in esso sparsi, qui elencati. Lelenco, il catalogo, l'assenza di ordine, «la vertigine della lista» è ciò che accomuna, ai fini di questo

${ }^{1}$ E. Edson - E. Savage-Smith, Medieval views of the cosmos: Picturing the Universe in the Christian and Islamic Middle Ages, Oxford, Bodleian Library-Oxford University, 2004, p. 9. 
intervento, $i$ due testi e ci permette di rilevare come i tecnicismi astrali nella loro criptica, a volte aspra ed impoetica formula, possano alimentare l'evocazione ${ }^{2}$. Nel Sonetto astrale:

Prevale il catalogo. Un raffinato collage di tecnicismi (rari alcuni, appartenenti al codice specialissimo dell'astronomia) compone in paratassi un sonetto che mette in scena un universo in moto ma non contemplato con sbigottimento, o ammirazione per l'artefice ${ }^{3}$.

La precisione del tecnicismo si fa qui suono, oggetto fonetico, che vibra quasi per osmosi con il lessico evocativo che scorre accanto ${ }^{4}$.

Non c'è artefice dunque, né relazione con l'umano, se non nel «vocativoesclamativo sbigottito» finale, che richiama Gozzano, e in cui si inserisce attraverso il possessivo (mie) l'unica «reazione personale del soggetto» allo spettacolo cosmico. Lontano dal pessimismo Leopardiano, forse più vicino al Pascoli astrale «ma senza voler rapportare, come fa Pascoli, l'umano alla vertigine degli abissi, e l'eternità del ruotare di corpi celesti all'effimera pulsazione di una vita terrena che scorre breve», Sanguineti:

È, come dev'essere, poeta del nostro tempo, tremendamente 'moderno'. Gli enormi progressi della scienza li conosciamo, sappiamo che non hanno svelato i misteri, la vastità dei cieli continua a sfidare l'immaginazione (sua, come quella dei più grandi astrofisici). Anche se sappiamo enormemente di più rispetto a quanto sapevano Dante, o Leopardi, o Pascoli, questo ha però altrettanto enormemente ingigantito il senso della fragilità della vita, la percezione di un destino che continua ad appendersi ai fragili fili di fantasiosi segni zodiacali ${ }^{5}$.

I progressi della scienza astronomica del Novecento, la trasformazione radicale dell'immaginario che l'ha accompagnata, la diffusione di immagini e di parole incide sulla lingua comune. La mutata visione del cosmo risemantizza parole antiche e ne mette in circolazione di nuove. I tecnicismi sembrano rompere il mito, spezzare la catena del discorso e riformulare lo spazio e l'immaginario ${ }^{6}$.

${ }^{2}$ U. Eco, La vertigine della lista, Milano, Bompiani, 2009.

${ }^{3}$ Riprendo di seguito alcuni passaggi del commento al sonetto contenuto in G.L. Beccaria, Sanguineti astrale, Torino, G. Giappichelli Ed., 2011, p. 14.

4 «Le difficili parole impoetiche del circo celeste, allineate come su uno schermo da un presentatore («ecco a voi...»), compongono versi che si dispongono per aggiunzioni, e come per gioco spettacolare $[. .$.$] , con un inconfondibile stile blob che tiene a braccetto il tecnico e l'alto$ lessico evocativo [...]. Lessico che rammemora l'illustre tradizione poetica [...]. Gli oggetti celesti sono nominati uno a uno [...]: oggetti celesti dai nomi precisi e, insieme, oggetti fonetici, perché la parola nasce parola». Ibid., pp. 14-16.

${ }^{5}$ Ibid., p. 19.

${ }^{6}$ Secondo Ricard P. Feymann, in Sei pezzi facili, l'astronomo cerca di cogliere il significato del «grande schema» di cui è parte e «saperne qualcosa non distrugge il mistero, perché la realtà 
Del resto, ad ogni crisi della scienza, corrisponde una crisi del linguaggio:

Nei momenti in cui la scienza vive stagioni fortemente rivoluzionarie (si pensi a Galileo o ad Einstein), essa tende ad avvicinarsi molto di più alle procedure della letteratura perché, volendo rovesciare verità sclerotizzate, fa vedere i fenomeni in modo straniante, con occhi diversi. Si pensi a tutti gli esperimenti mentali con cui Galileo cerca di abituare il lettore a pensare che la Terra si stia muovendo, aiutandolo a scrollarsi di dosso il pregiudizio che gli deriva dall'inganno dei sensi. Non solo, ma poiché nei periodi rivoluzionari gli scienziati innovatori non hanno in partenza prove sicure e oggettive di avere ragione, perché altrimenti le loro tesi sarebbero accolte senza discussione, essi tendono a impiegare anche i mezzi più tipicamente letterari con cui rendere più accattivante il discorso, che si arricchisce così di valori estetici. Per questo si deve convenire con Italo Calvino allorché ritiene che Galileo «meriterebbe d'esser famoso come felice inventore di metafore fantasiose quanto lo è come rigoroso ragionatore scientifico» ${ }^{7}$.

I tecnicismi citati nei due testi appartengono ad una nomenclatura specifica, certo, ma diffusa attraverso la divulgazione scientifica e quindi nota in gran parte anche ai non addetti ai lavori. Queste parole vivono oltre il ristretto ambito dell'astronomia? In quali forme? In questo intervento si realizza una campionatura degli usi traslati di bolide e bólido, se ne segue la vita fuori dall'astronomia, osservando quali tratti semantici accendano il traslato, in definitiva come le nuove conoscenze del cosmo entrino nella lingua. L'attenzione è volta alla stampa scritta. Senza pretese di esaustività, si realizza un sondaggio orientativo che permette di osservare fenomeni la cui estensione e applicazione possono essere misurate solo attraverso ulteriori studi e esplorazioni mirati nel tempo.

2.ANDATA E RITORNO DELLE METAFORE:VERSO LA LINGUA COMUNE E ALTRI LINGUAGGI SPECIALISTICI

È caratteristica dei linguaggi scientifici l'adozione di parole comuni alle quali si attribuiscono poi nuovi signifcati. Bertha Gutiérrez Rodilla, dopo aver segnalato il doppio movimento dal comune allo specialistico e viceversa, puntualizza:

è tanto più meravigliosa di quanto potesse immaginare nessun artista del passato! Perché i poeti oggi non ne parlano? Che uomini sono mai i poeti, che riescono a parlare di Giove pensandolo simile a un uomo, ma se è un'immensa sfera di metano e ammoniaca ammutoliscono?». Beccaria 2011: 16-17.

${ }^{7}$ A. Battistini, Le risorse conoscitive e estetiche della metafora, in «La metafora tra letteratura e scienza», Bari, Servizio Editoriale Universitario, 2006, pp. 19-37: pp. 34-35. 
Prácticamente en todos los dominios del conocimiento se da ese trasvase de terminología hacia la lengua estándar, aunque lógicamente es más fácil que se realice desde aquellos más próximos que desde los más lejanos de la realidad cotidiana ${ }^{8}$.

Forse l'astronomia è un settore avvertito come lontano dalla vita quotidiana. $\mathrm{Se}$, da un lato, si è indubbiamente persa quella familiarità con il cielo notturno caratteristica delle società rurali, oggi, con il grandissimo sviluppo della scienza astronomica e della divulgazione delle scoperte (si pensi alle immagini satellitari, ai sempre più numerosi musei delle scienza, ai planetari, al numero crescente di astrofili, etc.) si sta riformulando una familiarità con il cielo che, come si indicherà oltre, si lega in special modo alla cosmologia.

Eppure i corpi celesti sono stati a lungo ben presenti nella praxis umana e formano parte della nostra cultura. Scriveva Octavio Paz:

Cultura es aquello que el hombre usa, por ejemplo: el petróleo; y aquello que el hombre nombra, por ejemplo: una estrella. La Vía Láctea es parte de nuestra cultura; no es un valor de uso como el petróleo, pero es un conocimiento, un saber sobre el cielo y una imagen: fue un mito en la antigüedad y ahora es una metáfora que usamos diariamente 9 .

Il cielo stellato, visibile a occhio nudo, è stato per secoli percepito come parte integrante del panorama e della vita quotidiani. Grande orologio in movimento, segnale di cabiamenti meteorologici e del procedere delle ore nella notte, il cielo notturno scandiva con precisione il ritmo delle opere e i giorni in una società rurale ancora attiva fino alla metà del secolo scorso $^{10}$.

Nel caso di nomi propri di oggetti celesti, il riferimento fisico, sensibile, sul quale poggia il traslato ha caratteristiche peculiari. Se, infatti, il procedimento di associazione metaforica, in specie nella tassonomia popolare, si basa su un'equivalenza del tipo «x sta a $\mathrm{X}$ come y sta a Y» dove la caratteristica che permette il traslato può essere legata alla forma, al colore, al gusto, al tatto ossia a un dato tangibile del referente, nel caso dei referenti astrali tale dato tangibile è essenzialmente, anche se non esclusivamente, legato alla percezione a occhio nudo del fenomeno e alla sua relazione con il tempo. Studi precedenti su questo argomento hanno permesso di osservare come l'associazione metaforica alla base della denominazione di corpi celesti visibili a occhio nudo si fondi essenzialmente sulla relazione tra la visibilità dei corpi celesti di notte e il

8 B. Gutiérrez Rodilla, La ciencia empieza en la palabra. Análisis e bistoria del lenguaje científico, Barcelona, Península, 1998, p. 104.

9 A. Martín-Municio, "La metáfora en el lenguaje científico", Boletin de la Real Academia Española, Tomo 72, Cuaderno 256 (1992), pp. 221-250: p. 227.

${ }^{10} \mathrm{Nel}$ seguente paragrafo riprendo considerazioni generali sviluppate in P. Capponi, I nomi di Orione. Le parole dell'astronomia tra scienza e tradizione, Venezia, Marsilio, 2005. 
calcolo del tempo: cronologico, in relazione al procedere delle ore nella notte; meteorologico, in relazione alle condizioni del tempo e, infine, mitico (ciclico, eterno, eroico e sacro). Le tre forme di misurazione del tempo non si escludono a vicenda, bensí si integrano. Lo scorrere del tempo è colto nelle sue ricadute terrene: il tempo è rappresentato attraverso le attività che si svolgono in un certo periodo dell'anno o ora della giornata (si esprimerà attraverso l'indicazione del nome dello strumento usato, del mestiere, etc.) e sfuma spesso in un riferimento simbolico che attraverso i secoli e le religioni celebra santi ed eroi riproducendo nel cielo un pantheon pagano e cristiano. Nella tradizione colta, erudita, scritta, nell'iconografia antica di testi astrali il riferimento è al mito, agli eroi del mito. $\mathrm{Si}$ tratta di una tradizione conservata in testi che nei secoli si sono spostati tra Oriente e Occidente e che segna profondamente l'immaginario celeste ${ }^{11}$. Le costellazioni dell'emisfero australe ricordano invece la fiducia nella scienza e nelle conquiste della tecnica, proprie dellepoca in cui furono scoperte. Portano i nomi di strumenti della scienza e della tecnica, utili alla navigazione, alla misurazione.

La motivazione formale (che accompagna quella funzionale), sottesa al nome, cioè la forma che si attribuisce alla costellazione, è appiglio visivo, quasi aiuto mnemonico per fissare nel cielo figure che, pur del tutto aleatorie (uniscono astri lontani tra loro), presentano una straordinaria continuità nel tempo.

Sono poi i nomi antichi e classici delle costellazioni ad entare nella nomenclatura ufficiale delle 88 costellazioni stabilita dall'IAU (International Astronomical Union). La quantità di scoperte negli ultimi secoli ha reso infatti indispensabile un intervento da parte della comunità internazionale per stabilire criteri e norme per la denominazione del nuovo. Ho già avuto modo in altre occasioni di occuparmi del tema. Qui basti ricordare che i sistemi adottati per nominare morfologie planetarie e satelliti, ad esempio, tendono a stabilire una nomenclatura che oltre alle caratteristiche comuni ad altre nomenclature scientifiche (trasparenza, monoreferenzialità, assenza di riferimenti politici, religiosi, etc.) si distingue per la continuità con il passato attraverso il mito. Il mito è il punto d'unione tra tradizione e scienza. Il mito usato nella scienza quasi come filiera genealogica. Si stabiliscono aree semantiche alle quali si attinge. L'area semantica rimanda spesso all'appartenenza a un capostipite (si pensi ai nomi dei satelliti ad esempio) e, spesso, o, almeno, finché è possibile, la cronologia della scoperta segue, accompagna, la genealogia di dei ed eroi, ripercorre saghe mitiche.

11 Non sono poi mancati nella storia tentativi eruditi di cristianizzare il pantheon pagano riconducendolo a forme cristiane. Si pensi, per esempio, al Coelum stellatum Christianum di Julius Schiller, Augustae Vindelicorum: Praelo Andreae Apergeri, 1627. 
Nel corso del XX secolo poi, accanto alla tassonomia scientifica usata dagli addetti ai lavori, è aumentata notevolmente la circolazione di parole tecniche, la divulgazione del nuovo:

I linguaggi scientifci [...], e già l'abbiamo visto con Galilei, se da un lato ridefiniscono continuamente i loro sistemi di significati, dall'altro continuamente si pongono il problema di "agganciare" un pubblico che li realizzi nella storia e ciò è possibile solo attraverso i meccanismi evocativi di quella lingua comune da cui hanno preso le mosse ${ }^{12}$.

Louis Guilbert fu tra i primi ad occuparsene e segnalò parole che accompagnavano le nuove imprese dell'uomo e le nuove mete. Ricordare le parole di quegli anni riporta al clima di fiducia nella tecnica e nella tecnologia che rendeva possibile l'impresa, le prime missioni spaziali.

Lo ricordava già Gian Luigi Beccaria, nel 1973, nel suo I linguaggi settoriali in Italia, quando citava il caso dell'astronautica come esempio di passaggio dal vocabolario tecnico specializzato allo stadio di vocabolario di massa avvenuto tramite la stampa e citava:

orbitare, allunare, allunaggio, (1959; foggiati su atterrare-atterraggio, ammarareammaraggio), cosmonauta, astronauta (1961), sonda spaziale, propellente (1949), retro-razzo, pressurizzare (ingl. Pressurize); i giornali parlano con disinvoltura, piuttosto che del più trasparente rendez-vous, di docking, di spash-down dell' "Apollo" nel Pacifico, di "traiettoria translunare a ritorno libero", di modulo lunare, di razzovettore, di cabinamadre, di impatto (adattamento dell'ingl. Impact adoperato dai giornalisti che hanno descritto gli effetti della bomba di Hiroshima sulla "zona di impatto")13.

Bruno Migliorini ebbe modo di indagare la disputa tra i prefissoidi cosmo e astro nelle designazioni celesti, segno di una guerra fredda che si consumò anche nel cielo e nel cielo, con l'allunaggio dell'Apollo 11, ebbe uno dei suoi simboli. Particolarmente vincolato allo sviluppo della scienza del cielo è infatti il prefissoide cosmo- che, accanto alla ricca tradizione filosofica, trova, con lo sviluppo delle nuove tecnologie e l'avvio delle prime missioni spaziali, una stagione di rinnovata fioritura. Cosmonauta e cosmonave (il francese cosmonaute è del 1934) sono foggiati su astronave e astronauta. I composti con cosmo- sono preferiti dai sovietici, che hanno diffuso internazionalmente il loro Kosmonavt e Kosmodrom nonché la dilatazione di Kosmiceskij 'cosmico' nell'accezione di 'spaziale'. Rileggere le pagine di Bruno Migliorini in merito, sulla voce astronauta, rimanda al sapore e al clima dei primi viaggi spaziali: «Le recenti 188-189.

12 G. L. Beccaria (a cura di), I linguaggi settoriali in Italia, Milano, Bompiani, 1976, pp. 13 Ibid., p. 16. 
imprese spaziali hanno fatto comparire, accanto ad astronauta, il sinonimo cosmonauta: come tale è stato festeggiato Gagàrin in Russia e fuori, mentre per Shepard si sono adoperati i due nomi promiscuamente». Commenta Migliorini:

Anche se sia possibile fare una distinzione teorica fra astronauti e cosmonauti non direi che (almeno per ora) essa abbia una qualsiasi utilità pratica. La probabilità maggiore è perciò che essi rimangano ambedue come sinonimi indifferenziati. Se uno solo dovesse prevalere, i maggiori titoli li avrebbe astronauta, che ha un uso più vecchio (dal 1921 almeno), e confermato dall'esistenza di numerose società astronautiche nazionali ${ }^{14}$.

In questi casi si tratta di parole legate alla tecnica, alla tecnologia, alla techné che permette d'arrivare ad altri pianeti e mondi. Una volta entrate nella dinamica d'uso le parole tecniche, sia quelle che indicano i nuovi oggetti scoperti sia quelle che designano strumenti e apparecchi, si riattivano e si spostano all'interno della lingua, declinandosi in contesti diversi:

No es del todo infrecuente que términos originalmente neutros, desde el punto de vista de que estamos hablando, con el paso del tiempo vayan cargándose de una serie de connotaciones que, como decimos, no tenían en su inicio, comportándose en esto como el resto de las palabras [...]. Ese cambio en la neutralidad del término ocurre, en muchas ocasiones, cuando se produce su entrada desde el lenguaje científico en el lenguaje común ${ }^{15}$.

\section{Gutiérrez Rodilla cita in merito un brano di Foucault:}

¿A qué se debe que las palabras que, en su esencia primera, son nombres y designaciones y que se articulan de acuerdo con el análisis de la representación misma, puedan alejarse irresistiblemente de su significación original, adquirir un sentido cercano, más amplio o más limitado? Cambiar no solo de forma, sino también de extensión? ¿Adquirir nuevas sonoridades y también nuevos contenidos $[\ldots]$, [tanto que haya] palabras cuyo sentido no se recupera ya? ${ }^{36}$.

${ }^{14}$ B. Migliorini, Profili di parole, Torino, Le Monnier, 1968,pp.14-15.L'astronauta sembrerebbe avere una meta definita, una o più astri, mentre il cosmonauta navigherebbe senza chiara meta negli spazi extra-terrestri. Il significato di cosmo nel passato ha oscillato tra globo e universo, ambiguità condivisa con l'universo: «come ben si vede se si confronta l'Unione postale universale con la gravitazione universale». Conclude Migliorini: «Più increscioso sarebbe che i Russi finissero col chiamarsi cosmonauti e gli Statunitensi astronauti: in questa sia pur così parziale e precaria uniformità delle terminologie scientifiche greco-latine ogni separazione è un danno. Non basta che già democrazia e tante altre parole abbiano due significati quasi opposti al di qua e al di là della cortina di ferro?» (ivi). Cfr. P. Capponi, «Tra lingua dell' astronomia e lingua comune», in Cuadernos de Filología Italiana, 21, 2014, pp. 137-142.

${ }^{15}$ Gutiérrez Rodilla (1998: 93).

${ }^{16} \mathrm{Ivi}$. 


\section{BOLIDE/BÓLIDO: LA STORIA, L'IMMAGINARIO E LA PAROLA17}

Ogni fenomeno che accade nell'atmosfera è una meteora. Sono 'le cose sospese, sollevate', dal greco tà metéora (a sua volta da un derivato del verbo aírein 'sollevare' e dalla preposizione metá 'oltre'). Venti, turbini, cicloni, aurore boreali e arcobaleno, sono meteore (aeree i primi, luminose gli ultimi due) (DIR, s.v.).

In astronomia meteora è la scia luminosa che solca il cielo notturno «quando frammenti di materiali provenienti dallo spazio entrano nell'atmosfera terrestre, raggiungendovi per attrito alte temperature» (Gribbin 1998, s.v.) ${ }^{18}$. $\grave{E}$ anche detta stella cadente cui tradizionalmente si affidano desideri. Per l'impressione di fugacità che ne deriva si usa per indicare tutto ciò che risplende, fa scalpore per breve tempo e poi scompare (DIR, s.v.). «Poiché particelle di polvere e frammenti di roccia (risultanti dalla dissoluzione di comete o asteroidi) orbitano in sciami intorno al Sole, molte meteore si presentano in piogge meteoriche in certi momenti dell'anno, quando la Terra interseca l'orbita di uno di tali sciami» (Gribbin 1998, s.v.). Derivato da meteora è meteorite (maschile o femminile). Le meteoriti sono «frammenti di materiali rocciosi o metallici provenienti dallo spazio che, a differenza delle meteore, sono sopravvissuti al passaggio nell'atmosfera terrestre raggiungendo la superficie della Terra». Alla caduta di grandi meteoriti «sono stati ricondotti disastri ecologici che hanno causato estinzioni di massa di specie terrestri, come i dinosauri, scomparsi alla fine del Cretaceo. [...] Il peso delle meteoriti varia, da qualche decina di tonnellate a pochi grammi; sono state rinvenute circa una dozzina di meteoriti oltre le 10 tonnellate»(Gribbin 1998, s.v.) ${ }^{19}$. Le meteoriti sono anche detti bolidi o uranoliti.

L'immagine del bolide, nota dall'antichità e visibile ad occhio nudo, ha progressivamente acquisito una maggiore definizione, divulgazione, saldandosi, con il procedere della conoscenza, con paure antiche e nuove. Il referente, prima percepito come traccia luminosa nel cielo accompagnata da rumore, detonazioni, si fa immagine filmata, riproducibile e diffusa.

$\mathrm{Si}$ tratta di un fenomeno clamoroso che ben si presta a farsi notizia sulla stampa. Cito alcuni titoli recenti: Bolide straordinario sopra il Friuli («Coelum»,

${ }^{17}$ Per una prima esplorazione degli usi traslati nel corso del Novecento sono stati consultati i corpora CORIS/CODIS, DiaCORIS/CODIS, CREA e CORPE. Si è circoscritta l'analisi, dunque, agli usi scritti della lingua. Per lo spagnolo si è limitata l'analisi alla Spagna. L'attenzione si concentra poi sull'analisi delle occorrenze nell'ultimo quarto di secolo (1976-2000) nella stampa. La consultazione dei corpora italiani non sempre restituisce indicazioni specifiche sulla fonte da cui è estratto il termine.

${ }^{18} \mathrm{~J}$. Gribbin, Enciclopedia di astronomia e cosmologia, Milano, Garzanti, 1998.

19 Meteoroide è invece un «termine generico per designare gli oggetti che, incontrando la Terra, possono trasformarsi in meteore o in meteoriti. Un pezzo di roccia che cade su Marte è un meteoroide, non un meteorite. Fin tanto che un pezzo di roccia viaggia nello spazio può essere chiamato meteoroide o asteroide». Gribbin 1998, s.v. 
n. 174, p. 38); Palla di fuoco illumina la notte ("Corriere del Veneto», 3/09/2013); Meteoritos en España: así cayó el bólido que iluminó Madrid en julio de 2012 («E1 Mundo», 18/02/2012), España, iluminada por la bola de fuego más brillante jamás vista («ABC»,17/07/2012); Una bola de fuego cruza el cielo de Madrid y convierte la noche en día ( $\mathrm{ABC}$ »,15/04/2013)20. Il bolide più noto in Spagna è forse quello caduto il 10 febbraio 1896, così descritto in «La Vaguardia» dell'11 febbraio:

Oyóse un estampido sordo e intenso, seguido de otros muchos pero fuertes, parecidos al de una bala de cañón al pasar por lo alto de un piso. Estos ruidos fueron de duración extraordinaria, pues el fenómeno no duró menos de dos minutos. Mientras tanto producíanse trepidaciones de puertas, vidrieras y ventanas. Parece que se trata, sin embargo, del paso a través de la tierra de uno de estos cuerpos celestes, de origen poco conocido, tal vez restos de algún cometa, que en virtud de su inconcebible velocidad, de 40 a 60 kilómetros por segundo, al chocar con la capa atmosférica densa y comprimida se inflaman y estallan. («La Vanguardia», 11/02/1896: p. 5)

Allo schianto del bolide che 65 milioni di anni fa colpì la Terra dedica Telmo Pievani l'apertura di La fine del mondo (2012):

Il più grande spettacolo dopo il big bang cominciò senza preavviso. In unepoca primordiale che nessuno ricorda, un bolide roccioso di dieci chilometri di diametro si affacciò dallo spazio profondo e piombò in mezzo al mare, al largo della costa dello Yucatán. [...] Fu come se milioni di bombe atomiche scoppiassero in un sol colpo, deflagrando nella più potente esplosione di tutti i tempi [...] Nulla fu mai come prima. Più della metà delle specie, di ogni ordine e fattezza, dal plancton al dinosauro, non sopravvisse alla maledizione

${ }^{20}$ Si legge nel testo della notizia: «Una palla di fuoco ha illuminato per circa 5 secondi la scorsa notte i cieli del Nordest, fino a Modena e Ferrara. Si tratta di un bolide, ovvero una meteora ad alta luminosità, entrato in collisione con l'atmosfera a decine di chilometri d'altezza (circa 80) che frammentandosi ha originato un boato che ha svegliato di soprassalto centinaia di cittadini». In Spagna un grande bolide illuminò il cielo di Madrid nel luglio del 2012. In un articolo pubblicato su «E1 Mundo» del 18/02/2013 (T. Guerrero, Meteoritos en España: así cayó el bólido que iluminó Madrid en julio de 2012) si spiega che: «El 13 de julio de 2012 a las 2h 04m 51s (hora peninsular) apareció una enorme bola de fuego que iluminó durante cuatro segundos varias comunidades autónomas. Este fenómeno se produjo debido a la entrada en la atmósfera terrestre de una roca que surcaba el medio interplanetario. La roca viajaba a una velocidad estimada de unos 90.000 $\mathrm{km} /$ hora. El meteoroide, que según sus cálculos debía medir un metro de diámetro, estalló a unos 40 kilómetros de altura, provocando, según aseguraron numerosos testigos, un intenso destello que fue visto desde Andalucía, Castilla-La Mancha, Comunidad de Madrid, Extremadura, Murcia y la Comunidad Valenciana». Gli astronomi non diedero immediatamente notizia del fenomeno per evitare che i cazameteoritos li anticipassero nella raccolta di possibili meteoriti. Alejandro Sánchez de Miguel, astronomo del Dipartimento di Astrofisica dell'Università Complutense di Madrid, spiega la ragione del ritardo: «fue para evitar que los cazameteoritos recogieran posibles restos de rocas, ya que a partir de estas imágenes se puede localizar la zona en la que se produjo». 
piovuta dal cosmo. Perché il mondo tornasse a respirare, ci vollero migliaia, forse milioni, di anni ${ }^{21}$.

Oltre, nel capitolo dedicato al "disastro per cause esterne", ci ricorda che «siamo costantemente esposti a fenomeni fisici potenzialmente letali, e non soltanto terrestri» (Pievani 2012: 63-64). La storia è costellata da impatti di questo tipo e ancora ve ne saranno. I satelliti artificiali hanno individuato circa 150 crateri da impatto, alcuni di dimensioni considerevoli. Quando nel 1994 la cometa Shoemaker-Levy si infranse su Giove con la sequenza di 21 frammenti che si schiantavano sul gigante gassoso: «per la prima volta l'umanità assistette a uno spettacolo che è già accaduto, e potrebbe ancora accadere, anche sul nostro pianeta». Prosegue: «Erano gli anni in cui trovava conferma l'ipotesi dell'impatto di un asteoride come causa per spiegare l'estinzione di massa dei dinosauri. Tanto bastava per creare un nuovo genere catastrofico, suggellato anche da facili pellicole statunitensi come Deep Impact (1998) e Armageddon (1998)»²2. Nell'introduzione a "Come si comunica la scienza?» Yurij Castelfranchi e Nico Pitrelli insistono su come la scienza influenzi il vivere quotidiano:

Fornisce un sistema di simboli, concetti, valori, che ci aiutano a dare un senso alla realtà, a cercare risposte alle nostre domande, e, soprattutto, a formularne di nuove. Ecco perché la scienza è tra noi anche quando non è accompagnata dalla tecnologia. I concetti e le conoscenze, ma anche le metafore, gli stereotipi, le immagini che ci vengono dalla scienza e dalle sue rappresentazioni entrano a far parte dei sogni, delle emozioni, dei timori e delle speranze della nostra epoca $^{23}$.

\subsection{Bolide e bólido: migrazioni metaforiche della parola}

Una macchina veloce e potente è un bolide (Migliorini 1942, giá usato da Alfredo Panzini negli anni Venti), termine tratto dal vocabolario dell'astronomia (Marchi, 1828), dove la voce designa un corpo astrale che attraversa gli strati superiori dell'atmosfera diventando incandescente. $\grave{E}$ in origine voce dotta, latina, usata già da Plinio per indicare la 'meteora'. Rimanda a sua volta al greco dove vale 'oggetto lanciato'(dal verbo bàllein, lanciare'). In riferimento a 'persona corpulenta' è attestata nel 1965.

Nel vocabolario Treccani bolide è:

21 T. Pievani, La fine del mondo, Bologna, Il Mulino, 2012, pp. 7-8. Dopo aver descritto lo scenario, conclude: «Noi Homo sapiens, dunque, siamo figli di questa catastrofe orrenda. Dobbiamo essere grati a quel mostro letale di dieci chilometri di diametro che ha tagliato l'atmosfera e ha portato l'inferno sulla Terra. Dovremmo onorarlo nei secoli a venire, perché ha decretato la fine del mondo degli altri, e un nuovo inizio per chi proprio non se l'aspettava» (ibid., pp. 8-9).

22 Ibid., pp. 64-65.

23 Y. Castelfranchi, N. Pitrelli, Come si comunica la scienza, Bari, Laterza, 2007, p. X. 
Nome generico delle stelle cadenti o meteore che appaiono in cielo con scia brillante, larga e persistente, e, talora, scoppî e detonazioni: si tratta di blocchi e frammenti (generalmente piccoli) di materia metallica o pietrosa, che diventano luminosi nell'attraversare la parte più densa dell'atmosfera terrestre; con significato specifico, il termine indica la parte di tali blocchi che riesce a raggiungere la superficie terrestre, essendo allora sinon. di meteorite.

Parola antica, bolide è parola che attraversa il Novecento: nel periodo compreso tra il 1901 e il 1975 occorre 22 volte nel DiaCORIS/CODIS. Il $68.18 \%$ delle occorrenze è relativo alla stampa (quotidiani o periodici). Il resto delle occorrenze si distribuisce tra narrativa (18.18\%), saggistica (9.09\%) e miscellanea (9,09\%). Se consideriamo il periodo dal 1901 al 2001, osserviamo che è maggiormente presente nella prima metà del secolo (16 ossia 69.56\%) rispetto alla seconda (7 ossia $30.43 \%)^{24}$.

Se osserviamo la parola in contesto, l'aggetivazione ad essa riservata e la sua evoluzione nel tempo vediamo nel primo decennio del Novecento l'uso della parola nel ciclismo, per indicare la persona, non il mezzo. L'aggettivo umano (bolide umano) è usato in quattro occasioni sulla «Gazzetta dello Sport» da Armando Cougnet (che inventò e diresse il Giro d'Italia per circa quarant'anni) per riferirsi al ciclista Luigi Ganna, vincitore nel 1909 del primo Giro d'Italia.

In pezzi dal tono epico si legge:

Non è un uomo che corre: è una valanga umana che precipita, che balza, è un bolide umano lanciato lungo la via bianca che accieca, in un parossismo di forza cosciente e di furore. («La Gazzetta dello Sport», 1909: Luigi Ganna vince anche la quinta tappa. Ancora Ganna!)

E oltre:

Il gruppo dei fuggenti preme sui pedali in una pazza fuga che li difenda prima che il bolide arrivi. E i chilometri diminuiscono...e qualche testa si volge, dubitosa, sperando. Ahimè! Ecco il bolide che giunge, schiumando polvere dai raggi infuocati, lanciando fiamme dagli occhi. (Ibid.)

Le occorrenze successive ci portano agli anni Trenta, dove la parola cambia contesto e compare preceduta dai verbi deviare (2), respingere, bloccare, segnali tutti dell'appartenenza al linguaggio calcistico. Cito un solo esempio:

Se gli spagnoli, più che mai vivi e intraprendenti, poterono stampare un pallone su un palo della rete di Combi, un altro palo respinse un bolide di Guaita. ( $« \mathrm{La}$ Gazzetta dello Sport», 1934: Zamora saracinesca quasi inespugnabile)

${ }^{24} \mathrm{La}$ scelta dei periodi di tempo considerati nell'analisi dei corpora è stata determinata dalle caratteristiche proprie di consultazione di ciascun corpus e dall'esigenza di ritagliare lassi di tempo confrontabili tra le due lingue. 
Una sola occorrenza ci porta fuori dal contesto sportivo: giungere come un bolide, espressione usata in riferimento alla notizia del patto di non aggressione russo-tedesco. Tra i significati figurati della voce si trova infatti passare, precipitarsi, piombare come un bolide, con la velocità di un bolide, a grandissima velocità (Treccani, s.v.). In questo caso, il traslato poggia sull'idea di sorpresa e paura, sgomento, minaccia:

A Parigi e a Londra l'annuncio del patto di non aggressione russo-tedesco è giunto come un bolide. La sorpresa è stata pari allo sbigottimento: tanto che qualche giornale non ha esitato a scrivere che si trattava di una notizia falsa. La verità è che, sfasciatasi la chiave di volta del piano di accerchiamento, nuovi sviluppi si profilano nella situazione internazionale. («Il Corriere della Sera», 1939: La storia cammina)

Negli anni Cinquanta Indro Montanelli su «Il Nuovo Corriere della Sera» scrive un pezzo dal titolo Nuvolari in cui ricorda la celebre vittoria del mantovano volante nel Gran Premio di Germania del 1935. Sconfisse a sorpresa la grande favorita, la Mercedes Benz, davanti a un pubblico di ufficiali tedeschi e davanti a Adolf Hitler che seguiva dagli spalti il Gran Premio. Gli pneumatici dell'automobile condotta da von Brauchitsch cedettero e vinse l'Alfa Romeo di Nuvolari. Scrive Montanelli:

Nuvolari, di fronte a lui, naufragava tra le fronde di lauro della corona che strascicava per terra le sue foglie, mentre il meccanico veniva trasportato allospedale, dove doveva restare sei mesi, e qualche migliaio di occhi azzurri fissavano sbarrati e increduli i resti del bolide rosso sui quali l'Italiano aveva tagliato in testa a tutti lo striscione di arrivo. («Il Nuovo Corriere della Sera», 1950: Nuvolari)

Sempre in ambito sportivo, Gianni Brera commenta l'eliminazione dell'Italia dai mondiali del 1958 e nella sua prosa ritroviamo l'epicità guerresca del linguaggio calcistico:

Cush arrestò comodissimamente la palla, anzi, ebbe l'esitazione caratteristica, dopo il controllo, di chi teme di essere in fuori gioco: poi subito esplose un bolide che Bugatti fu molto bravo, anzi eroico a parare: ma sulla respinta -inevitabile- del nostro sfortunato portiere, non un difensore azzurro; Cush ebbe ancora tutto il tempo di riprendere e sparare a rete a colpo sicuro. («Il Giorno», 1958: Eliminati)

Si rintraccia poi il termine nella cronaca politica: è Leo Longanesi a firmare su «Il Borghese», da lui fondato, un articolo dedicato a Alcide De Gasperi: 
Chi aveva da trattare con lui, non si vedeva, no, comparire dinanzi il solito uomo politico italiano di formazione o filodrammatica o avvocatesca, arrivato nella politica estera come un bolide, e abituato a mettere a posto l'Europa nei comizi; ma un uomo che, bene o male, aveva veduto l'Europa nei suoi giorni di splendore davvero "unitario”, e ci credeva ... («Il Borghese», 1954: Alcide de Gasperi)

Negli anni Sessanta Dino Buzzati scrive sul «Corriere della Sera» un pezzo sul disatro del Vajont e lo intitola Natura crudele. L'articolo inizia così:

Come ricostruire con la mente ciò che è accaduto, la frana, lo schiantamento delle rupi, il crollo, la cateratta di macigni e di terra nel lago? E l'onda spaventosa, da cataclisma biblico, che è lievitata gonfiandosi come un immenso dorso di balena, ha scavalcato il bordo della diga, è precipitata a picco giù nel burrone avventandosi, terrificante bolide di schiume, verso i paesi addormentati? E il tonfo nel lago, il tremito della terra, lo scroscio nell'abisso, il ruggito folle dell'acqua impazzita, il frastuono della rovina totale, coro di boati, stridori, rimbombi, cigolii, scrosci, urla, gemiti, rantoli, invocazioni, pianti? E il silenzio alla fine, quel funesto silenzio di quando l'irreparabile è compiuto, il silenzio stesso che c’è nelle tombe? («Il Corriere della Sera», 1963: Natura crudele)

Ancora in ambito sportivo, il termine è usato in riferimento al motociclismo, in particolare alle note sfide tra Stanley Michael Bailey Hailwood e Giacomo Agostini. Qui ritroviamo quell'aggettivo, umano, prima applicato al ciclismo:

Hailwood, costretto a forzare l'andatura per non perdere il contatto con quella specie di bolide umano che gli era passato accanto e che era subito schizzato via, senza neppure dargli il tempo di tirare il fiato, ha dovuto sottoporre la sua Honda a uno sforzo troppo gravoso e il motore della quattro cilindri, come era già avvenuto altre volte, ha ceduto di colpo al settimo giro. («Il Corriere della Sera", 1966: Agostini volava quando Hailwood si è ritirato)

La parola filtra dunque nel linguaggio giornalistico attraverso la cronaca sportiva per segnalare prestazioni eccezionali in riferimento alla velocità e alla potenza, prima nel ciclismo, poi nel calcio, nell'automobilismo e nel motociclismo ${ }^{25}$. Il contesto d'uso è legato ad un tipo di prosa dal colore epico,

25 In Fraseología y metáfora: aspectos tipológicos y cognitivos, Eva María Iñesta Mena e Antonio Pamies Bertrán così si riferiscono alla velocidad: «Como la velocidad es por definición espacio dividido por tiempo, la transferencia conceptual se realiza desde y hacia el concepto de movimiento». Può essere espressa attraverso riferimenti a un fenomeno atmosferico (como un rayo, con la velocidad del rayo, como el viento, come un fulmine), ma anche con un oggetto, in particolare associando la persona veloce ad una macchina ( $a$ todo gas, a toda máquina, a toda pastilla, come un razzo), rimanda anche all'area del conflitto, ai colpi e alle armi con cui vengono sparati, lanciati (como una bala, como una flecha). Rimandi al fenomeno atmosferico, alla macchina e al lancio si ritrovano in varia misura nella definizione dello spettro semantico di bolide. E. M. Iñesta Mena 
iperbolico, celebrativo dell'impresa. Quando non appartiene al contesto sportivo, il termine è usato per insistere sul tratto della sorpresa, dell'evento inatteso, di solito con segno negativo. Il tratto euforico legato all'impresa sportiva è qui capovolto, ciò che arriva inatteso è temuto, minaccioso.

Se concentriamo l'attenzione sugli usi della parola nell'ultimo quarto di secolo attraverso il CORIS/CODIS rileviamo 34 occorrenze (29 su quotidiani, 2 su periodici, 3 in supplementi). Possiamo osservare che l'aggettivazione che accompagna il sostantivo rimanda per lo più all'automobilismo (nuovo bolide -2-, bolide nero, rosso) e al calcio (bolide angolato). Non potendo risalire alla datazione dei documenti, analizziamo le occorrenze del termine in base alla tipologia individuata dall'analisi dei casi precedenti, quindi /velocità/ e /potenza/ (in relazione a calcio, ciclismo, automobilismo, motociclismo), /sorpresa/, /minaccia/. Dall'analisi emerge che il tratto che sostiene il traslato è soprattutto la velocità, l'eccellenza delle prestazioni. Scompaiono sorpresa e minaccia. Il termine pare stabilizzarsi negli ambiti dello sport, prevalentemente nel calcio (16 occorrenze), quindi nell'automobilismo (12) e nel motociclismo (1) ${ }^{26}$. Il riferimento alla velocità si sfuma in un più generico riferimento all'"eccezionalità delle prestazioni del mezzo". Scompare il ciclismo e ricorre l'aggettivo nuovo ad indicare i progressi della tecnologia nella Formula 1. Pare assottigliarsi il peso del fattore umano: prevalgono il veicolo e i progressi della tecnica che rendono possibile il miglioramento costante, il superamento continuo dei limiti.

Il termine compare poi in contesto scientifico, astronomico, in due articoli firmati da Luigi Bignami su «Il Corriere della Sera - Scienza». Del primo, dal titolo Terremoto misterioso in Australia. Forse è stato un bolide celeste, incuriosisce la presenza della specificazione, celeste, che, oltre a dare coloritura al titolo, è forse indice del fatto che il termine bolide, sia sempre più usato fuori

- A. Pamies Bertrán, Fraseología y metáfora: aspectos tipológicos y cognitivos, Granada, Método ediciones, 2002: pp. 199-208.

${ }^{26}$ Solo in un caso, in un articolo che tratta dello sbarco degli Italiani a Durazzo, nell'aprile del 1997, la parola è usata in riferimento a un autoblindo: «Dal buio sbuca Arco, autoblindo pesante della classe Centauro, un bolide da 28 tonnellate che con le sue enormi ruote corre a 120 chilometri all'ora». Non sono inclusi nella categoria automobilismo due riferimenti ironici all'automobile veloce (non alla Formula 1) che cito di seguito: «Tutti pronti? Si parte. Dovete rassegnarvi, il cane non amerà mai il vostro bolide. Salire in macchina per lui è come per voi fare un giro su una navicella spaziale». In una lettera all'allora ministro dell'Interno Rosa Russo Jervolino in merito ai limiti di velocità, il termine è usato per richiamare allusivamente e ironicamente lo status sociale associato al bolide, alla macchina potente (feeling da bolide per la Millemiglia): «Caro Ministro Jervolino, si fa un gran discutere sui limiti di velocità, sulla necessità di un minimo di coscienza sulla strada; a parole siamo tutti angioletti con il volante a mo' di aureola, ma qui come si fa! È estate, l'autostrada è intasata dai Tir, hai voglia di guidare e di sentirti in vacanza e magari sei su una Audi TT roadster, cuore turbo e feeling da bolide per la Millemiglia. Forse l'abito non fa il monaco, ma l'automobile cambia l'uoomo [sic], eccome». Analogamente, non è incluso nella categoria motociclismo il riferimento generico a moto potente in un articolo di cronaca relativo ad un incidente stradale («Corriere della Sera», 1997: Franziska motociclista pentita: "Ora andrò veloce solo in vasca"). 
dall'ambito scientifico. Del secondo, tratto da «Il Corriere della Sera - Scienza» del 23 febbraio 1997, Il ritiro dei mari avviò l'estinzione, riporto la conclusione notando come l'autore ricorra a asteroide e meteorite nel corpo dell'articolo, mentre sceglie bolide per la chiusura, quando si insiste sull'eccezionalità dell'evento, cedendo quasi ad un tono più narrativo, come se bolide (accompagnato qui dal verbo impattò) portasse con se un alone evocativo più marcato, consono appunto alla chiusura del testo:

Comunque, l'importanza della caduta dell'asteroide sulla Terra è stata messa in luce recentemente con maggiore dettaglio da altri due ricercatori americani, Peter Schultz e Steven D'Hondt della Rhode University, i quali hanno pubblicato sulla rivista Geology i risultati di uno studio che dimostrerebbe che l'angolo con il quale l'asteroide colpì la Terra 65 milioni di anni fa fu il peggiore che mai potesse scegliere un oggetto celeste per colpire il pianeta. Alcune simulazioni dimostrerebbero che il bolide impattò con la Terra con un angolo compreso tra 20 gradi e 30 gradi sollevando la maggior quantità di materiale che si possa produrre da un evento simile. («Il Corriere della Sera Scienza», 23.02.1997, Il ritiro dei mari avviò l'estinzione)

Da notare, nelle cronache sportive di calcio, l'insistenza su quel tono epico guerresco (già rilevato nell'analisi dei testi precedenti) che pare rimandare dal bolide alla balistica: «lascia partire un bolide», «da vero cecchino con un bolide dal limite», «sventa un bolide di Baldini, «Pira sfodera un bolide che colpisce l'incrocio dei pali», «Paolo Marchi che spara un bolide dalla distanza».

Non emergono invece sulla stampa casi che riportino il termine all'accezione scherzosa di uomo grosso e robusto. Ne troviamo un caso invece tra le 86 occorrenze restituite da una ricerca in DiaCORIS/CODIS:

Da questi tortelli è nata la mia piccola tragedia. Una sera mia sorella, la piccola e fragile creatura di mia madre, portò a cena il suo ragazzo, un bolide maleducato che non riesco a sopportare. (V. Cultrera, Panico, Rimini, Guaraldi, 1992)

Se ora osserviamo, sulla scorta delle osservazioni sviluppate in merito agli usi traslati in italiano, gli usi di bólido in spagnolo noteremo un uso meno insistito nella stampa e usi meno variegati.

La parola bólido, si legge nel DRAE, deriva dal latino bolis, - ̌̆dis, a sua volta dal greco $\beta$ oic is, arma da lanciare, lancio e ha attualmente le seguenti due accezioni:

1. m. Vehículo automóvil que alcanza extraordinaria velocidad, especialmente el que participa en carreras.

2. m. Meteor. Masa de materia cósmica de dimensiones apreciables a simple vista que, con la apariencia de un globo inflamado, atraviesa rápidamente la atmósfera y suele estallar y dividirse en pedazos 
Una ricerca del lemma in Mapa de diccionarios (che raccoglie sei edizioni del dizionario accademico -1780, 1817, 1884, 1925, 1992 y 2001-) ci permette di osservare che la voce è presente dal 1884 (assente nelle edizioni precedenti) e solo nell'accezione meteorologica: «m. Meteor. Especie de meteoro ígneo que cruza el espacio con gran velocidad». La voce si mantiene nel 1925, ma con una definizione rivista, più precisa: «m. Meteor, Masa mineral de dimensión apreciable a simple vista que, a manera de globo inflamado, atraviesa rápidamente la atmósfera y suele estallar en pedazos» e con l'aggiunta dell'accezione figurata: «m. fig. Vehículo automóvil que alcanza extraordinaria velocidad, especialmente el que participa en carreras». Entrambe le definizioni si mantengono nelle edizioni successive, fino ad oggi. L'accezione figurata è dunque attestata a partire dal 1925.

Dalla ricerca delle occorrenze del termine nel CORDE (limitando l'indagine agli anni 1901-1975 e alla Spagna) osserviamo che i 37 casi appartengono tutti alla categoria libros. Non sono registrati usi nella stampa. Tuttavia, osservando i documenti che la ricerca restituisce, notiamo tra gli autori la presenza di noti divulgatori scientifici: al 1905 risale la pubblicazione di Ciencia popular, volume che raccoglie un centinaio di articoli di divulgazione scientifica scritti da José Echegaray, pubblicati su «El Imparcial» e «El Liberal» e raccolti in volume dagli Ingenieros de Caminos dopo che l'autore, nel 1904, ricevette il premio Nobel. Delle 37 occorrenze, 6 appartengono a questo volume. La prosa ricorda forse un altro grande divulgatore, Camille Flammarion. Cito alcuni passaggi:

Ese pedazo del bólido, que á capricho manejamos entre nuestros dedos, ha estado donde no podemos estar nunca; habrá visto, si hubiera podido ver, lo que jamás veremos; podría contarnos, si supiera contar, misterios que serán para nosotros siempre impenetrables; vino á nosotros, que nosotros no hubiéramos podido ir á él; en cada una de sus partecillas hay guardado un secreto, que será eterno silencio; allá arriba, entre los otros, estaba hasta hace poco, y hoy está aquí.

Siglos y siglos en regiones heladas; llegó á la tierra y se incendió, por algo será.

Ganas dan de decirle á cualquiera de esos trozos de bólido:

"Masa inerte, yo no te puedo subir como tu has bajado: pero mi pensamiento y mi espíritu, suben más que tu bajas, y también se incendian."

Lo cual será verdad, con una condición, con la de que no vivamos siempre á ras de tierra. (J. Echegaray, Ciencia popular, Madrid: Imprenta de Hijos de J.A. García, 1904: 614-615)27.

27 Poco prima scriveva: «La Tierra con sus palpitaciones geológicas y con sus palpitaciones humanas, el bólido con sus granillos de hierro, estúpidamente inerte, girando y girando sin pensar, sin querer, sin desear. Hace tres días, las inmensidades del espacio les separaban. ¿Qué español 
Troviamo poi un altro noto divulgatore scientificio, José Comas y Solá, che in un passaggio ricorda il bolide del 1896 di Madrid²8:

Fácilmente se comprende, según lo dicho, que los aerolitos deben ser de tamaño muy pequeño; $y$, en efecto, rara vez alcanzan dimensiones considerables. El bólido que cayó en Madrid el 10 de febrero de 1896 se dividió en varios fragmentos, el mayor de los cuales tiene solamente el tamaño de una naranja pequeña. (J. Comas y Solá, Astronomia I y II, Madrid, Espasa-Calpe, 1932, I, p. 378)

Tra le occorrenze nella categoria libros rientrano poi opere volte all'insegnamento ${ }^{29}$. Se proseguiamo nella ricognizione degli usi traslati del termine in questi contesti, non giornalistici, ritroviamo poi alcuni sensi figurati noti, comuni all'italiano, come nell'espressione como un bólido. Ne cito alcuni estratti dal CORPE: «Exclamación que cae como un bólido en sus meditaciones» (Miguel de Unamuno, Amor y pedagogia); «Mal sonaba en los oídos de Ansúrez el tristísimo nombre de la que, arrojada de los aires y cayendo sobre él como un

sospechaba la existencia del bólido allá en las profundidades de la extensión? Y aunque la masa meteórica, que hoy tomó vecindad entre nosotros, hubiera tenido pensamiento y hubiera podido sospechar algo, nunca hubiera podido sospechar la existencia de una provincia española. Pues, de pronto, la tierra pasa por los bordes del anillo, ó por las regiones donde el bólido caminaba, y lo que no había sucedido en miles de siglos, sucede en solo un punto y en un instante solo. La tierra arranca al bólido de su órbita, lo llama, á sí, lo atrae, lo hunde en su atmósfera, lo inflama; ya es masa de fuego, ya estalló. Y hoy andan en manos de ciudadanos españoles pedazos de bólido, que andaban en lo profundo de la extensión á millones de kilómetros, hace poquísimo tiempo. No vienen esos pedazos que entre nuestras manos tenemos, ni de debajo de tierra, ni de otro lado de los mares, ni del seno de una del éter, de las regiones planetarias, de esas regiones en donde nosotros no podemos penetrar, que para nosotros representan lo imposible». J. Echegaray, Ciencia popular, Madrid, Imprenta de Hijos de J.A. García, 1904, pp. 613-614.

28 Alla sua firma si devono 3 occorrenze del termine. Così Ramón Gómes de la Serna in Automoribundia (1948) ricorda il bolide del 1896: «Pero cuál era entre esas vaguedades la fórmula de la felicidad de ese cacareado año de 1896? [... Fué el año del bólido que cayó el 10 de febrero y que sublimó al aerolito, llegando a pensarse con temor si se aficionaría el cielo a arrojar esos proyectiles». Ramón Gómes de la Serna, Automoribundia, Buenos Aires, Editorial Sudamericana, 1948, pp. 106-107. E così Arturo Barea, nella trilogia La forja de un rebelde (1951): «Cuando estalló el polvorín en Carabanchel, todos los cristales se cayeron rotos por la escalera abajo. Era muy pequeño, pero me acuerdo que mi madre me bajó en brazos a la calle, corriendo porque no sabía lo que pasaba. La gente estaba por entonces muy asustada, porque hacía muy pocos años que había caído un gran bólido cerca de Madrid. Luego hubo una erupción enorme en un volcán que hay en Italia, que se llama El Vesubio, y además vino el cometa Halley». A. Barea, La forja de un rebelde, Losada, Buenos Aires, 1951, par. 1.

${ }^{29} \mathrm{Si}$ veda J. Benejam, La escuela práctica: obra destinada a promover la enseñanza primaria moderna mediante ejercicios (1904-1905), Universidad de Alicante, Alicante, 2003: par. 10: «Se discurrirá sobre los bólidos y aerolitos, que son fragmentos de bólidos, advirtiendo que la caída de un bólido se manifiesta con luz y explosión o varias explosiones, cayendo a veces con tanta fuerza que se hunden en el terreno y se quedan estacionados. Si se tocan estas piedras, luego de haber caído, se advierte que queman». 
bólido, fue coscorrón y donativo de la Providencia» (Benito Pérez Galdós, La vuelta al mundo en la Numancia) ${ }^{30}$.

Da segnalare invece un uso poco consueto in spagnolo che si rileva in Caza menor di Elena Soriano, dove la parola indica la massa di un corpo inerte:

El inmenso, blando bólido de su corpachón hizo vibrar todo el barandal de la escalera al apoyarse impulsivamente sobre él. Flora subía ya, desalada, preguntando qué sucedía ${ }^{31}$. (E. Soriano, Caza menor, Madrid, CastaliaInstituto de la Mujer, 1992 (1951), p. 380)

Se passiamo in rassegna la presenza della parola bólido nella stampa spagnola dal 1976 al 2001, in base ai dati del CREA otteniamo 24 occorrenze su un totale di 39. Di queste, la maggior parte si riferisce a quotidiani (23) e solo una è contenuta in rivista. La distribuzione per area tematica, restituisce i seguenti risultati: deportes (11), tecnología (4), actualidad (2), astronomia (2), arte y cultura (1), política (1), tauromaquia (1). Prevale nettamente la categoria deportes seguita da tecnología.

Come nei casi precedenti, occorre osservare le parole in contesto per chiarirne il significato e ridisegnare la mappa dei riferimenti traslati. Nella sezione deportes il rimando è esclusivamente all'automobilismo, in articoli con riferimento a Airton Senna («E1 País», 01/10/1989: El brasileño logró su $40^{\circ}$ 'pole position' en el Campeonato Mundial, 1 occorrenza), a Niki Lauda («El País», 5/05/1976: Niki Lauda, el jinete de los "cavallinos rampantes", 5 occorrenze), al nuovo Wolf wrl («E1 Pais», 10/11/1976: El Wolf WRL, nuevo bólido de fórmula-1, 3 occorrenze); all'incidente in cui morì il pilota francese Patrick Depailler («El pais», 2/10/1980: Patrick Depailler se mató en Nockenheim, 1 occorrenza). È poi qui annoverata la seguente occorrenza del termine: «Como en un bólido, por el cerebro del Bernabéu recorrerán recuerdos imborrables: las remontadas europeas ante el Anderlecht, el Inter o el Borussia; los pases inimaginables, [...] y los goles sin huecos» («El Mundo», 15/06/1995: Fútbol. Visperas del partido de despedida de Emilio Butragueño), dove la parola rimanda alla velocità con cui si rincorrono i ricordi.

All'automobile possono essere ricondotte un totale di 18 occorrenze (85.7\%). Ai riferimenti contenuti nella sezione deportes si aggiungono le 4 occorrenze registrate in tecnología che si riferiscono al superamento della barriera del suono da parte del pilota Andy Green a bordo del Thrust («El País», 14/10/1997: El bólido británico 'Thrust' rompe la barrera del sonido). L'inclusione nella categoria

30 M. de Unamuno, Amor y pedagogia, Madrid, Turner, 1995 (1902), p. 192; B. Pérez Galdós, La vuelta al mundo en la Numancia, Biblioteca virtual Miguel de Cervantes, Universidad de Alicante (Alicante), 2002 (1906), p. 11.

31 Oltre, il termine ricorre ancora: «Pero un buen día, sucedió algo de otra índole: Josefa irrumpió en la cocinilla, sede de la Rufa, con la violencia de un bólido, y comenzó a insultarla a gritos». E. Soriano, Caza menor, Madrid, Castalia-Instituto de la Mujer, 1992 (1951), p. 399. 
tauromaquia è dovuto al fatto che il termine è citato in un pezzo in cui si ricorda la fascinazione prodotta sullo spettatore dalle prodezze dei piloti. La parola bolide è usata per rievocare la vertiginosa velocità, il vorticare delle macchine sul circuito, con alone epico. Da notare l'uso dell'aggettivo automovilístico:

El estoicismo de cientos de personas inmóviles para ver pasar ante sus ojos vertiginosamente una motocicleta o un bólido automovilístico («El País», 01/06/1984, Un juego de arte y de inteligencia)

I riferimenti della categoria actualidad rimandano anche'essi all'automobilismo, all'incidente durante le qualifiche per il Gran Premio del Belgio del 1982, a Lovanio, in cui morì Gilles Villeneuve e sono entrambi contenuti in «ABC», 14/05/1982: El bólido se desintegró en tres trágicos "saltos"32.

Nella categoria arte, invece, con bólido teledirigido ci si riferisce ai giochi per bambini, alle macchinine da corsa. Si tratta qui di una critica, di un esempio di giocattolo vuoto, la cui funzione si esaurisce nella prestazione che può fornire. Un giocattolo che non resta, non crea relazione, ma svela la sua essenza e in essa si esaurisce.

Igualmente, el cohete que anda con el sonido de la voz o el bólido teledirigido, raramente serán utilizados, tras averiarse, para otros destinos fuera del programa que el juguete traía marcado. («El País», 03/01/1981: Estética) ${ }^{33}$

Bólido (nel testo accostato a cohete, citato poco prima) esprime dunque il reclamo, l'eccezionalità che si rivela effimera, subito svelata dal meccanismo che la rende possibile, il telecomando e il suono della voce.

Nel seguente caso il contesto è politico e il riferimento è al fatto che una parte politica non possa sfoderare tutta la potenza:

Parece como si el potente bólido nacionalista se encontrara circulando en una noche de cerrada niebla y fuera incapaz de ver lo que hay detrás de cada curva («La Vanguardia», 15/11/1994: Una semana decisiva) ${ }^{34}$.

32 Troviamo poi due riferimenti all'astronomia nella rivista «Pléyades», 52, (2000): párr. 37 e in «I@c.noticias», 50 (2001): Leónidas 2001 en Australia.

${ }^{33}$ Bólido, così come bolide del resto, ha avuto una certa fortuna nella commercializzazione di macchine e giochi di simulazione di corse automobilistiche. Cito alcuni esempi: bólido clásico è un gioco di simulazione di corsa di macchine online, bólido Penélope è il nome di un modello di maacchinina, Bólido Campeón un gioco di costruzione di automobile (Lego).

${ }^{34}$ Il testo prosegue nella metafora del percorso, del procedere politico, come lungo una strada, facendo riferimento al «carro de la gobernabilidad»: «En estas circunstancias todo es posible. Incluso, que ante el enorme lío que tiene delante Jordi Pujol, se tenga la tentación de ir soltando amarras del carro de la gobernabilidad y se responsabilice de ello a Narcís Serra y a Pasqual Maragall» (Ibid.). 
E proprio ad una riflessione sulla velocità, sull'area semantica che la esprime, interviene Álex Grijelmo, facendo riferimento al valore euforico del verbo acelerar (e in climax ascendente cita: «nos gusta que se acelere una obra, que acelere un ciclista, o un piloto al volante de su bólido»):

El concepto "acelera" bucea también en los significados positivos, porque nos gusta que se acelere una obra, que acelere un ciclista, o un piloto al volante de su bólido, buscamos un coche con buena aceleración, y estamos dispuestos a perdonar a alguien que se equivocó en alguna decisión personal o profesional por culpa de que iba muy acelerado. Se activa así en el cerebro un concepto positivo y, aunque el prefijo "des" lo modifique ante nuestra percepción consciente, su perfume se habrá derramado también con la activación involuntaria de la palabra y sus sentidos. (A. Grijelmo, La seducción de las palabras, Madrid, Taurus, 2001, p. 134)

La seconda occorrenza dallo stesso testo serve quale esempio funzionale ad un' argomentazione sul genere delle parole e la loro relazione con il cambiamento sociale. Qui la parola bólido indica il nuovo, l'innovazione (último bólido) quasi a risemantizzare una parola, coche, il cui significato cambia con i cambiamenti della tecnica e della società:

Es decir, el genérico masculino seguirá existiendo, como la palabra "mensaje" pervive pese a las transformaciones de su significado a tenor de los cambios en la sociedad; igual que la palabra "coche" que identificó a aquéllos arrastrados por los caballos sirve ahora para designar al último bólido de los circuitos (Ibid.).

Da segnalare, come curiosità, anche se non si riferiscono alla stampa, le occorrenze incluse nella categoria salud, che rimandano al ritmo di vita eccessivamente veloce, frenetico: «Total, que voy todo el día de bólido» (A. Enríquez Soriano, Estrés. Cómo aprender en la encrucijada, Olaya ediciones, 1997). La stessa espressione, ir de bólido, ricorre poco oltre: «Ahora bien, practicar un ejercicio físico, nada tiene que ver con ir corriendo a buscar el pan, el periódico, ir de bólido a coger el bus, o para dejar a los niños en el cole antes de la reunión, ¿نerdad?» (Ibid., p. 188). Il tratto alla base del traslato è dunque la /velocità/ come qualità superiore alla norma e positiva (Deportes y Tecnología) o, più raramente, negativa (Salud).

In conclusione, la progressiva restrizione semantica, tecnica della parola non impedisce il traslato. Gli usi traslati di cui il sostantivo si fa portatore in entrambe le lingue ben esprimono quella tensione tra denotazione e connotazone, tra tecnico e comune che accompagna la storia della lingua in genere e che trova applicazione anche in un settore del lessico, quello astronomico, che pare 
particolarmente distante dalla quotidianità ${ }^{35}$. La parola conserva il proprio potere allusivo in entrambe le lingue con un grado d'estensione diverso in base ai contesti e alla storia. Riflettendo sull'uso di espressioni metaforiche nella scienza, Giovanni Valiati ricordava che forse «ci dovremmo stupire di parlare continuamente in poesia senza accorgercene». Le sue parole riportano alla riflessione sul movimento pendolare della lingua, tra restrizione tecnica e allusività, oscillazione che sotiene la stratificazione nel tempo dei significati:

Il linguaggio tecnico/scientifico, non meno del linguaggio volgare, è pieno di frasi e di espressioni metaforiche che, pur avendo cessato, pel lungo uso, di richiamare l'immagine che suggerivano originariamente, non hanno perduta la capacità di indurci ad attribuire ai fatti che esse descrivono tutte le proprietà dell'immagine a cui esse si riferiscono...al contrario di quel personaggio di Molière che si stupiva di aver sempre parlato in prosa senza saperlo, noi ci dovremmo stupire di parlare continuamente in poesia senza accorgercene ${ }^{36}$.

\section{BIBLIOGRAFIA}

Altieri Biagi, M.L., L'avventura della mente, Napoli, Morano Editore, 1990.

Barea, A., La forja de un rebelde, Losada, Buenos Aires, 1951.

Battistini, A., «Le risorse conoscitive e estetiche della metafora», en La metafora tra letteratura e scienza, Bari, Servizio Editoriale Universitario, 2006, pp. 19-37.

Beccaria, G.L., Sanguineti astrale, Torino, G. Giappicchelli Ed., 2011.

Beccaria, G.L. (a cura di), I linguaggi settoriali in Italia, Milano, Bompiani, 1976.

BenejAM, J., La escuela práctica: obra destinada a promover la enseñanza primaria moderna mediante ejercicios (1904-1905), Universidad de Alicante, Alicante, 2003.

Capponi, P., I nomi di Orione. Le parole dell'astronomia tra scienza e tradizione, Venezia, Marsilio, 2005.

CApponi, P., «Tra lingua dell' astronomia e lingua comune», en Cuadernos de Filología italiana, 21, 2014, pp. 137-151.

Castelfranchi, Y. - Pitrelli, N., Come si comunica la scienza, Bari, Laterza, 2007.

Comas y Solá, J., Astronomía I y II, Madrid, Espasa-Calpe, 1932.

CORDE - Real Academia Española: Banco de datos (CORDE) [en línea]. Corpus diacrónico del español. 〈http://www.rae.es>

CORIS/CODIS - Università di Bologna, CORpus di Italiano Scritto contemporaneo / COrpus Dinamico dell'Italiano Scritto <http://corpora.dslo.unibo.it>

35 Nella premessa a Linguaggi specialistici in italiano, Riccardo Gualdo e Stefano Telve rilevano come la presenza della terminologia specialistica nella lingua comune, sia evidente «in quelle discipline o in quei campi del sapere che intrattengono rapporti più intensi con la vita quotidiana» e citano le scienze biomediche, il linguaggio giuridico e la terminologia tecnica legata alle nuove tecnologie. Linguaggi specialistici in italiano, Roma, Carocci, 2012, p. 11.

${ }^{36} \mathrm{G}$. Vailati, Alcune osservazioni sulle question di parole nella storia della scienza e della cultura, Torino 1899, pp. 34-35 cit. in M. L. Altieri Biagi, L'avventura della mente, Napoli, Morano Editore, 1990, p. 380. 
CREA - Real Academia Española: Banco de datos (CREA) [en línea]. Corpus de referencia del español actual. <http://www.rae.es>

DEI - Battisti, C. - Alessio, C., DEI:Dizionario etimologico italiano, Firenze, Barbera, 1950-1957.

Deli - Cortelazzo, M. - Zolli, P., Dizionario Etimologico della Lingua Italiana, Bologna, Zanichelli, 1979-1988.

Unamuno, M. de, Amor y pedagogia, Madrid, Turner, 1995.

DIR - Gianni Angelo: Dizionario Italiano Ragionato, Firenze, D’Anna, 1987.

DiaCORIS - Università di Bologna, DiaCORIS, CORrpus Diacronico dell'Italiano Scritto <http://corpora.dslo.unibo.it>

DRAE - Real Academia Española, Diccionario de la lengua española, 22. ${ }^{a}$ ed. Madrid: Espasa, 2001.

Echegaray, J., Ciencia popular, Madrid, Imprenta de Hijos de J.A. García, 1904.

Eco, U., La vertigine della lista, Milano, Bompiani, 2009.

Edson, E. - Savage-Smith, E., Medieval views of the cosmos, Picturing the Universe in the Christian and Islamic Middle Ages, Oxford, Bodleian Library-Oxford University, 2004.

Enríquez Soriano, A., Estrés. Cómo aprender en la encrucijada, Madrid, Olaya ediciones, 1997.

Gómes de la Serna, R., Automoribundia, Buenos Aires, Editorial Sudamericana, 1948.

Gribbin, J., Enciclopedia di astronomia e cosmologia, Milano, Garzanti, 1998.

Grijelmo, A., La seducción de las palabras, Madrid, Taurus, 2001.

Gualdo, R. - Telve, S., Linguaggi specialistici dell'italiano, Roma, Carocci, 2012.

Gutiér rez Rodilla, B., La ciencia empieza en la palabra. Análisis e historia del lenguaje cientifico, Barcelona, Península, 1998.

Iñesta Mena, E.M. - Pamies Bertrán, A., Fraseología y metáfora: aspectos tipológicos y cognitivos, Granada, Método ediciones, 2002.

Martín-Municio, A., "La metáfora en el lenguaje ciéntifico", en Boletín de la Real Academia Española, Tomo 72, Cuaderno 256 (1992), pp. 221-250.

Migliorini, B., Profili di parole, Torino, Le Monnier, 1968.

Pérez Galdós, B., La vuelta al mundo en la Numancia, Biblioteca virtual Miguel de Cervantes, Universidad de Alicante (Alicante), 2002 (1906).

Pievani, T., La fine del mondo, Bologna, Il Mulino, 2012.

Soriano, E., Caza menor, Madrid, Castalia-Instituto de la Mujer, 1992 (1951). 
